\title{
Improving Linear Projects Georeferencing to Create Digital Models Using UAV Imagery
}

\author{
Amr M. Elsheshtawy ${ }^{1,2^{*}}$, and Larisa A. Gavrilova ${ }^{2}$ \\ ${ }^{1}$ Civil Engineering Department, Faculty of Engineering in Cairo, Al-Azhar University, Egypt \\ ${ }^{2}$ State University of Land Use Planning, Moscow, Russia
}

\begin{abstract}
Global Positioning System (GPS) on Unmanned Aerial Vehicles (UAV) platform relies on Micro Electro Mechanical Systems (MEMS) technology with a precision of $10 \mathrm{~m}$ at shooting time at UAV camera stations positions. Nonetheless, obstacles to the GPS signal at the finest flight altitude can prevent accurate camera stations positions retrieval. In this research, three different georeferencing techniques were compared with geometric precision. The first is Direct Georeferencing (DG), which mainly depends on using Navigation GPS onboard without using any Ground Control Points (GCPs). The second is Indirect Georeferencing (IG), which mainly depends on three GCPs used to assist Aero-Triangulation (AT). The third is Modified technique depends on the same three GCPs used in the second method and enhanced location of camera stations usage of the Linear Relation Model (LR Model). The study area was in the south of the Moscow Region, Russia. Three- imaging strips have been taken using the DJI PHANTOM 4 PRO UAV. The accuracy assessment was carried out using image-derived coordinates and checkpoints (CPs) residuals. This study emphasizes that the Modified methodology using enhanced camera stations positions gave better accuracy than using the drone GPS camera stations positions.
\end{abstract}

Keywords: UAV, Photogrammetry, Linear projects, Direct Georeferencing, Indirect Georeferencing, Aero-Triangulation, RTK-GNSS, DSM

\section{Introduction}

Developed countries are increasingly facing high maintenance costs of aging linear infrastructure, such as highways for transportation. The growth of the motor industry and subsequent economic development have produced a market for cleaner, better-performing, less congested highways [1]. High accuracy of information such as location, elevation, and feature information and good road alignment design in linear projects is very critical [2]. Because of payload limitations, regularly UAV's Inertial Measurements Units (IMU) depend

* Corresponding author: amrshesht82@gmail.com 
on MEMS innovation, whose demeanor information is as of now still of insufficient quality, for the most part in yaw [3]. The low-cost methodology used to produce accurate photogrammetric products (DEM, Orthophoto, 3D model ... etc.) by optimizing georeferencing with a limited number of GCPs and MEMS [4].

Economic sensors thus typically have resolution SR between $1 \mathrm{~cm}$ in RGB sensors and $10 \mathrm{~cm}$ in multi-spectral sensors. A problem with this ultra- SR is that it typically exceeds the precision of direct Exterior Orientation Parameters (EOPs) for evaluating individual images at the exposure time of the sensor. A particular method of assessing EOPs at the time of presentation requires an incorrect georeferencing of the corresponding orthophotomosaic or an error that generally exceeds the pixel dimension, at least. The receiver rate of position measurements per second $(\mathrm{Hz})$ is critical for sampling a moving object's position; for example, a $10 \mathrm{~Hz}$ Global Navigation Satellite System (GNSS) receiver samples one position per a half meter at a velocity of $5 \mathrm{~m} / \mathrm{s}$. On the other hand, a receiver may operate in code or carrier-phase mode, the latter is typically more reliable in the area, since the receiver may discern the oscillation of the sinusoidal wave, where the signal code is stored. This solution can be achieved expediently using a single-frequency receiver. Additionally, a dualfrequency differential GNSS (DGNSS) receiver may be used to improve the final performance. A DGNSS is capable of reading two carrier-phases at two wavelengths (typically L1/L2) and ignoring atmospheric effects (differential measurement) induced signal delays, as mentioned by [5]. A solution for determining the parameters of the exterior orientation (Space resection) based on algorithms based on genetic evolution. This space resection optimization model can be implemented with or without redundancy and doesn't require linearization [6].

The accuracy of the traditional UAVs Direct Georeferencing depends on the efficiency of the GNSS receiver; for example, in the precision code, the positioning error can reach 0.77 $\mathrm{m}$, and in the carrier phase L1/L2, the error can reach $0.01 \mathrm{~m}$ (Colomina and Molina 2014). Studies use an SR $<0.1 \mathrm{~m}$ for imaging. A classic accurate georeferencing technique for aerial imagery, known as Indirect Georeferencing, is the use of GCPs to assist Aero-Triangulation and achieve accuracies of $2 \mathrm{~cm}$, tested using Independent Checkpoints (ICPs) [7]. Since 1990, Post-Processing Kinematic (PPK) DG solution is a commonly used approach in airborne platforms [8]. Real-Time Kinematic (RTK) georeferencing is generally not needed in environmental and topographic mapping projects. However, it is important to get as accurate an image positioning as possible. This can be done by post-processing the central coordinates of the sensor at the exposure point, taking into account the trajectory of the UAV, the location of the sensor at the trigger [9]. Focus on improving data on the RTK-GNSS elevations as one of the critical means of assessing the number of earthworks. The principle of this development using the linear relation model is to build and compare many models from chosen points using Level and RTK-GNSS data to enhance the remaining RTK-GNSS data $[10,11]$.

Reconstructed from the UAV images, the 3D point cloud will obtain disaster information from various aspects of the artifacts involved. This usually requires moves including coarse match, thick match, modification, and estimation of the point cloud. To accomplish these moves, we need a lot of time. All the above approaches are applied, in the commercial software, such as PhotoScan (http://www.agisoft.com)

In this study, the geometric accuracy of three different georeferencing techniques was compared. The first is direct georeferencing that mainly depends on using onboard Navigation GPS without using any GCPs. The second is indirect georeferencing, which mainly depends on using three GCPs to support Aero-Triangulation. The third is a modified technique that mainly depends on enhanced camera stations positions by using the LR model and the same three GCPs which used in the second technique. The accuracy assessment was 
done using the residuals of image-derived coordinates and checkpoints, which have welldetermined surveyed coordinates on specific targets.

\section{Materials and method}

\subsection{Study area and data sources}

The study was carried out at the GORNOYE scientific and educational base of the State University of land use planning, located in the south of the Moscow Region. The imagery area is $900 \mathrm{~m} \times 90 \mathrm{~m}$.

Aerial imagery was carried out using DJI PHANTOM 4 PRO UAV (Figure 1). The threeimaging strips were taken using the DJI PHANTOM 4 PRO UAV, and 502 images were obtained. Flight lines were 900 meters in length. The end lap and side lab were about $80 \%$, each strip contains about 167 images. The Flight altitude was about 30 meters. The spatial resolution was about $1 \mathrm{~cm}$. The study used $34 \mathrm{GCPs}$, evenly distributed as groups throughout the study area. Each group consisted of about three GCPs and the groups were distributed every 50-100 meters in the length direction of the project $(900 \mathrm{~m})$. The distances between points in each group of GCPs were about 20 meters in the width direction of the project 90 $\mathrm{m})$. The three GCPs, which were used in methods two and three in georeferencing, are on the left side of the study area as shown in figure 2. Due to a larger percentage of overlap, these three GCPs were located in ten images inside the three strips. Camera calibration parameters are illustrated in table 1. RTK-GNSS, Trimble R4 dual-frequency GPS receivers were used to collect GCPs coordinates. The photogrammetric software Agisoft PhotoScan Professional was used for the photogrammetric data processing.

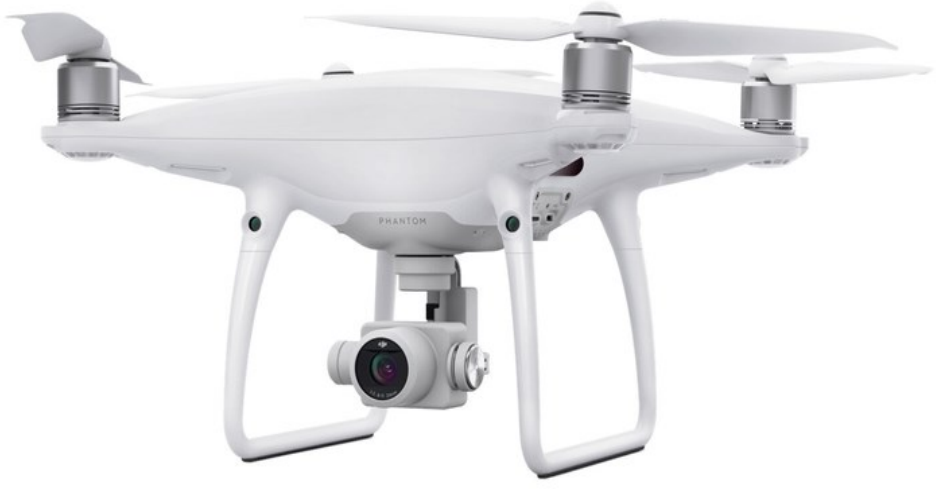

Fig. 1. DJI PHANTOM 4 PRO.

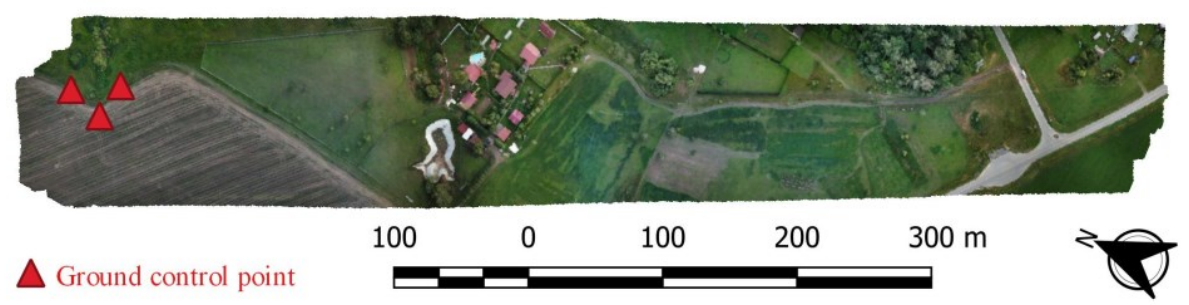

Fig. 2. The study area and the distribution of GCPs which used in georeferencing in methods 2 and 3 . 
Table 1. Camera calibration parameters.

\begin{tabular}{|l|l|}
\hline Camera Name & FC6310 $(8.8 \mathrm{~mm})$ \\
\hline \multirow{2}{*}{ Focal length } & $8.8 \mathrm{~mm}$ \\
\hline \multirow{4}{*}{ Principal Distance } & Width $=4864$ pixels \\
\cline { 2 - 2 } & pixel width $=0.0026 \mathrm{~mm}$ \\
\cline { 2 - 2 } & Height $=3648$ pixels \\
\cline { 2 - 2 } & pixel width $=0.0026 \mathrm{~mm}$ \\
\hline \multirow{3}{*}{ Coefficients of Radial Distortion } & $\mathrm{c}=9.5861 \mathrm{~mm}$ \\
\cline { 2 - 2 } & $\mathrm{x}_{0}=-0.0413 \mathrm{~mm}$ \\
\cline { 2 - 2 } & $\mathrm{y}_{0}=-0.0783 \mathrm{~mm}$ \\
\cline { 2 - 2 } Coefficients of Decentering Distortion & $\mathrm{K} 1=5.83450 \mathrm{e}-005$ \\
\cline { 2 - 2 } & $\mathrm{K} 2=1.12129 \mathrm{e}-006$ \\
\cline { 2 - 2 } & $\mathrm{K} 3=-1.18117 \mathrm{e}-008$ \\
\cline { 2 - 2 } & $\mathrm{P} 1=1.42270 \mathrm{e}-004$ \\
\hline
\end{tabular}

\subsection{Methods}

The various methods for studying the comparison of three UAV georeferencing techniques are shown in table 2 . In that, methods only camera stations positions (Xo, Yo, and Zo) were used in georeferencing and the three orientation angles were not used. In addition, different numbers of GCPs were used and the rest of the points were used as checkpoints.

Table 2. Parameters of the four UAV georeferencing techniques.

\begin{tabular}{|c|c|c|c|c|c|c|c|c|c|}
\hline \multirow[t]{2}{*}{ 是 } & \multirow[t]{2}{*}{ 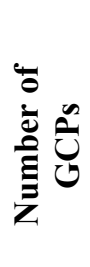 } & \multirow{2}{*}{ 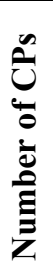 } & \multicolumn{3}{|c|}{$\begin{array}{c}\text { Camera } \\
\text { stations } \\
\text { positions } \\
\text { by drone GPS }\end{array}$} & \multicolumn{3}{|c|}{$\begin{array}{c}\text { Enhanced } \\
\text { camera } \\
\text { stations } \\
\text { positions } \\
\text { by modeling }\end{array}$} & \multirow[t]{2}{*}{ Remarks } \\
\hline & & & $\mathbf{X}_{\mathbf{0}}$ & $\mathbf{Y}_{\mathbf{0}}$ & $\mathbf{Z}_{\mathbf{0}}$ & $\mathbf{X}_{\mathbf{0}}$ & $\mathbf{Y}_{\mathbf{0}}$ & $\mathbf{Z}_{\mathbf{0}}$ & \\
\hline 1 & 0 & 34 & + & + & + & - & - & - & Direct Georeferencing \\
\hline 2 & 3 & 31 & + & + & + & - & - & - & Indirect Georeferencing \\
\hline 3 & 3 & 31 & - & - & - & + & + & + & Modified technique \\
\hline 4 & 34 & 0 & + & + & + & - & - & - & Reference method \\
\hline
\end{tabular}

From table 2 it can be seen that the direct georeferencing technique (method 1) uses only data of camera stations positions obtained by drone GPS and all 34 GCPs were used as CPs. On the other hand, indirect georeferencing technique (method 4) all 34 GCPs were used in georeferencing. The indirect georeferencing technique (method 2) used camera stations positions by drone GPS plus only three GCPs that were located on the left side of the block in georeferencing and the other 31 GCPs used as CPs. However, the modified technique used the same 3 GCPs in addition to the enhanced camera stations positions by LR model in georeferencing and other 31 GCPs used as CPs. Flowchart to show the three georeferencing techniques for UAV linear projects are shown in figure 3. 


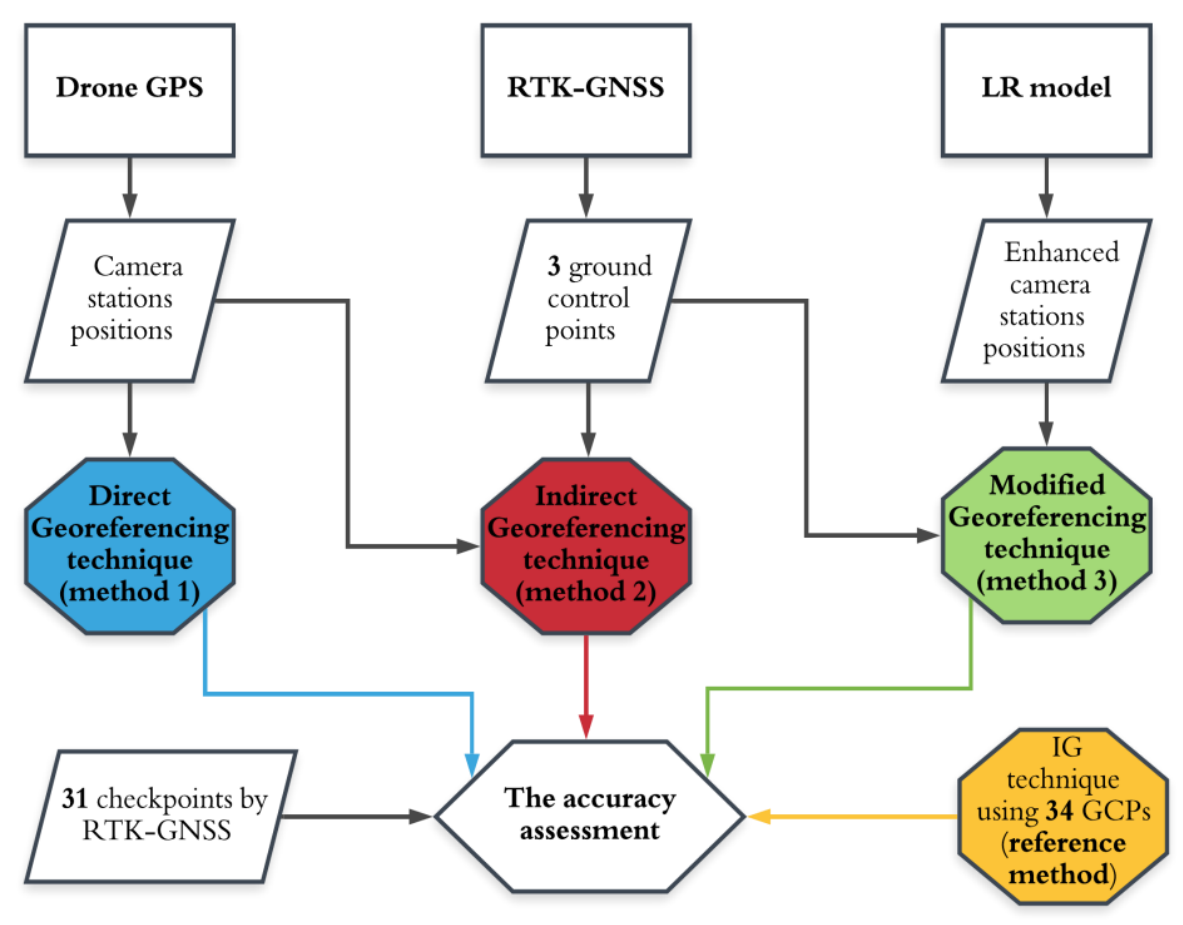

Fig. 3. Flowchart of the three-georeferencing techniques for UAV linear projects.

Methodology of enhancement camera stations positions obtained by drone GPS usage of LR model includes data acquisition task, determination of correlation between the auxiliary data by drone GPS and computed camera stations positions through initial georeferencing using a little number of GCPs in a small part of the project. The correlation in specified images are images that only contained the GCPs that were used in initial georeferencing.

The LR model contains two steps:

The first step of the LR model is to calculate the camera coordinates of images (XRi, YRi, ZRi) that have the three GCPs through initial georeferencing. In this research, ten images have the three GCPs. Equation No.1 has been used to find the mean value of the differences (DXav, DYav, DZav) between XRi computed through initial georeferencing and XGi obtained by drone GPS for the ten images that only contained the GCPs.

Where:

$$
\mathrm{DXav}=\frac{\sum_{\mathrm{i}=1}^{\mathrm{n}} \mathrm{DXi}}{\mathrm{n}}
$$

DXi - The differences between XGi and XRi (DXi = XGi - XRi).

XGi - Camera station position X of image $i$ obtained by drone GPS (X GPS).

$\mathrm{XRi}$ - Camera station position $\mathrm{X}$ of image $i$ computed through initial georeferencing (X reference).

$\mathrm{n}$ - Number of images.

In the second step, equation No.2 has been used to calculate the new Camera stations coordinates $(\mathrm{XNi}, \mathrm{YNi}, \mathrm{ZNi})$ using the mean value of the differences (DXav, DYav, DZav) obtained by equation No1.

Where:

$$
\mathrm{XNi}=\mathrm{XGi}-\mathrm{DXav}
$$

$\mathrm{XNi}$ - Generated Camera station position X of image $i$ for the rest of images (X new). 
XGi - Camera station position X of image $i$ obtained by drone GPS (X GPS).

Similar equations can be used for Camera station $\mathrm{Y}$ and $\mathrm{Z}$ coordinates.

The computed photo centers coordinates (XRi, YRi, ZRi) for the specified image and generated photo centers coordinates $(\mathrm{XNi}, \mathrm{YNi}, \mathrm{ZNi})$ using the LR model for the rest of the project images are used in final georeferencing for all UAV images.

The accuracy assessment was done using the residuals of checkpoints as follows:

$$
\mathrm{VXi}=\mathrm{XCi}-\mathrm{XGi}
$$

(3)

Where:

VXi is X residual of point $\mathrm{i}$.

$\mathrm{XCi}$ is $\mathrm{X}$ coordinate of point $i$ computed from adjustment ( $\mathrm{X}$ computed).

$\mathrm{XGi}$ is $\mathrm{X}$ reference coordinate of point $i$ from field survey observations (X reference).

In addition, the Root Mean Square Error of $X$ residuals Mx was calculated as follows.

$$
\mathrm{Mx}=\sqrt{\frac{\sum_{\mathrm{i}=1}^{\mathrm{n}}(\mathrm{VXi})^{2}}{\mathrm{n}}}
$$

Where $\mathrm{n}$ is the number of checkpoints.

Similar equations were used for $\mathrm{Y}$ and $\mathrm{Z}$ coordinates.

When Mx, is RMSE of X, My is RMSE of Y, and Mz is RMSE of Z. Therefore, Error is the position error can be calculated as follows.

$$
\text { Error }=\sqrt{(\mathrm{Mx})^{2}+(\mathrm{My})^{2}+(\mathrm{Mz})^{2}}
$$

\section{Results and discussion}

\subsection{Accuracy of the three methods compared to the reference method}

When testing the accuracy of the four methods 1, 2, 3, and 4. Figure 4 shows the errors of the various methods

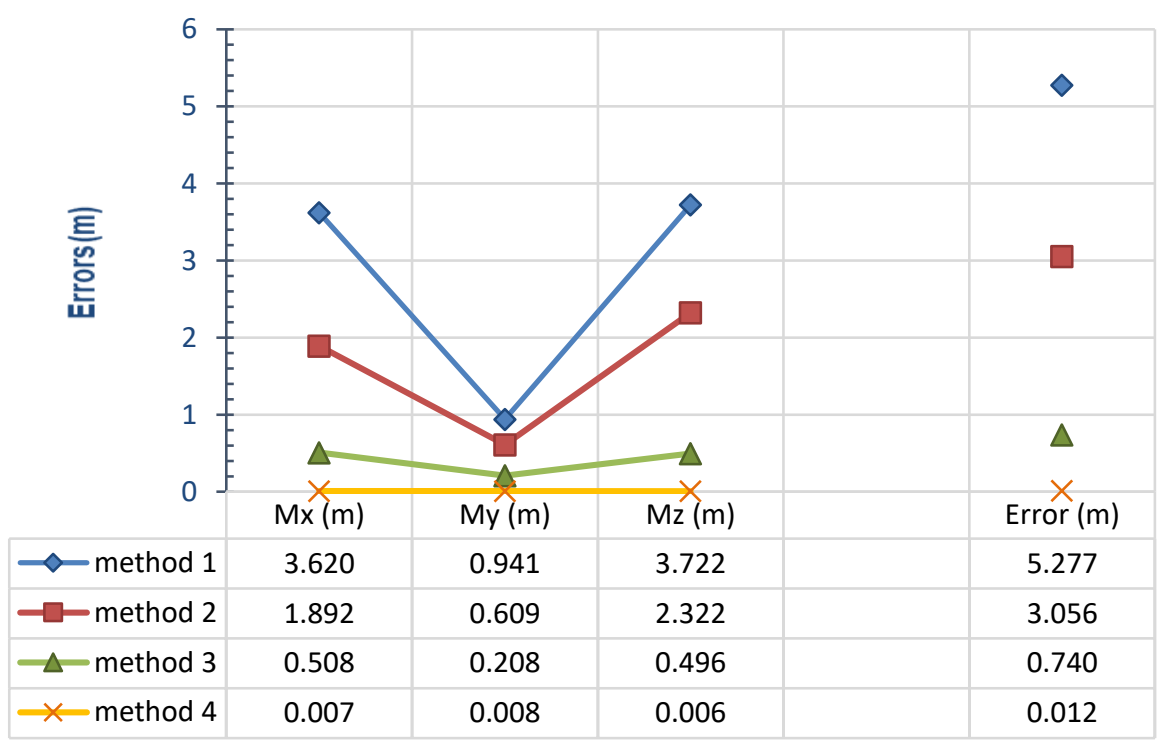

Fig. 4. Errors in method 1, method 2, method 3, and method 4. 
It can be seen in figure 4 according to the values of $\mathrm{Mx}, \mathrm{My}, \mathrm{Mz}$, and Error that the Direct Georeferencing technique without using GCPs (method 1) is the worst case, while the Indirect Georeferencing technique using $36 \mathrm{GCPs}$ (method 4) is the best. Hence, method 4 is the reference result for accuracy among all methods. Furthermore, When using in georeferencing 3 GCPs on the edge of the linear project and enhanced camera stations positions by LR model (method 3 ) is better than using the same 3 GCPs with drone GPS camera stations positions (method 2).

\subsection{Accuracy of the enhanced camera stations positions by LR model}

When comparing drone GPS camera stations positions and computed camera stations positions through georeferencing in method 4 as reference data, table 3 shows the descriptive statistics of differences between computed data and GPS data.

Table 3. Descriptive statistics of camera stations positions differences between computed data in method 4 and drone GPS data used in method 1.

\begin{tabular}{|c|c|c|c|}
\hline & $\mathbf{D X}_{\mathbf{0}}(\mathbf{m})$ & $\mathbf{D Y}_{\mathbf{o}}(\mathbf{m})$ & $\mathbf{D Z}_{\mathbf{0}}(\mathbf{m})$ \\
\hline Average & -5.64 & -1.75 & 3.66 \\
\hline Max. & -4.02 & 2.10 & 7.03 \\
\hline Min. & -8.82 & -6.67 & -1.67 \\
\hline RMSE & 5.70 & 2.62 & 4.17 \\
\hline
\end{tabular}

When comparing computed camera stations positions through georeferencing in method 4 as reference data, and the enhanced camera stations positions by LR model, which were used in method 3, the descriptive statistics of differences between computed data and enhanced data are shown in table 4.

Table 4. Descriptive statistics of camera stations positions differences between computed data in method 4 and enhanced data used in method 3.

\begin{tabular}{|c|c|c|c|}
\hline & $\mathbf{D X}_{\mathbf{0}}(\mathbf{m})$ & $\mathbf{D Y}_{\mathbf{0}}(\mathbf{m})$ & $\mathbf{D Z}_{\mathbf{0}}(\mathbf{m})$ \\
\hline Average & 0.44 & 0.53 & -0.04 \\
\hline Max. & 2.03 & 4.33 & 3.35 \\
\hline Min. & -2.77 & -4.43 & -5.36 \\
\hline RMSE & 0.95 & 2.01 & 2.03 \\
\hline
\end{tabular}

From the previous results in tables 3 and 4, it is clear that the enhanced camera stations positions which were used in method 3 are more accurate than drone GPS data that were used in method 1 according to the differences between the computed data in method 4 and them.

\subsection{Accuracy of the three methods along with the linear project}

In projects that GCPs would not be distributed all over the project like inaccessible areas or in disaster areas or to decrease the cost, or for any other reason else, the GCPs will be available on the edge of the linear project. It is important to know the accuracy of all parts along with the project from the beginning near the GCPs to the end of the project. Therefore, table 5 illustrates the errors of the various methods along with the UAV linear project. 
Table 5. The errors of the various methods along with the UAV linear project.

\begin{tabular}{|l|c|c|c|c|c|c|c|c|c|c|}
\hline $\begin{array}{l}\text { Numbers of Checkpoints or } \\
\text { GCPs in each group }\end{array}$ & 3 & 3 & 3 & 2 & 4 & 5 & 4 & 4 & 3 & 3 \\
\hline $\begin{array}{l}\text { Distance from beginning } \\
\text { of the project (m) }\end{array}$ & 100 & 200 & 300 & 400 & 450 & 500 & 600 & 700 & 750 & 800 \\
\hline $\begin{array}{l}\text { DG technique method 1 } \\
\text { errors (m) }\end{array}$ & $\mathbf{6 . 7}$ & $\mathbf{5 . 8}$ & $\mathbf{5 . 4}$ & $\mathbf{4 . 1}$ & $\mathbf{5 . 2}$ & $\mathbf{4 . 8}$ & $\mathbf{4 . 6}$ & $\mathbf{4 . 3}$ & $\mathbf{5 . 6}$ & $\mathbf{6 . 1}$ \\
\hline $\begin{array}{l}\text { IG technique method 2 } \\
\text { errors (m) }\end{array}$ & $\mathbf{0 . 0}$ & $\mathbf{0 . 2}$ & $\mathbf{0 . 4}$ & $\mathbf{0 . 7}$ & $\mathbf{2 . 1}$ & $\mathbf{2 . 6}$ & $\mathbf{2 . 1}$ & $\mathbf{2 . 6}$ & $\mathbf{3 . 9}$ & $\mathbf{3 . 4}$ \\
\hline $\begin{array}{l}\text { Modified technique } \\
\text { method 3 errors (m) }\end{array}$ & $\mathbf{0 . 0}$ & $\mathbf{0 . 1}$ & $\mathbf{0 . 2}$ & $\mathbf{0 . 4}$ & $\mathbf{0 . 6}$ & $\mathbf{0 . 7}$ & $\mathbf{0 . 9}$ & $\mathbf{1 . 0}$ & $\mathbf{0 . 9}$ & $\mathbf{0 . 9}$ \\
\hline
\end{tabular}

The comparison of the various methods was done using the average checkpoint errors in each group along with the project, which was distributed in sequence from the beginning to the end of the UAV linear project. Figure 5 shows the comparison of the various methods along with the UAV linear project. The location of the 3 GCPs (48-2, 50, and 51) which were used in georeferencing in methods 2 and 3 was $100 \mathrm{~m}$ away from the start of the project.

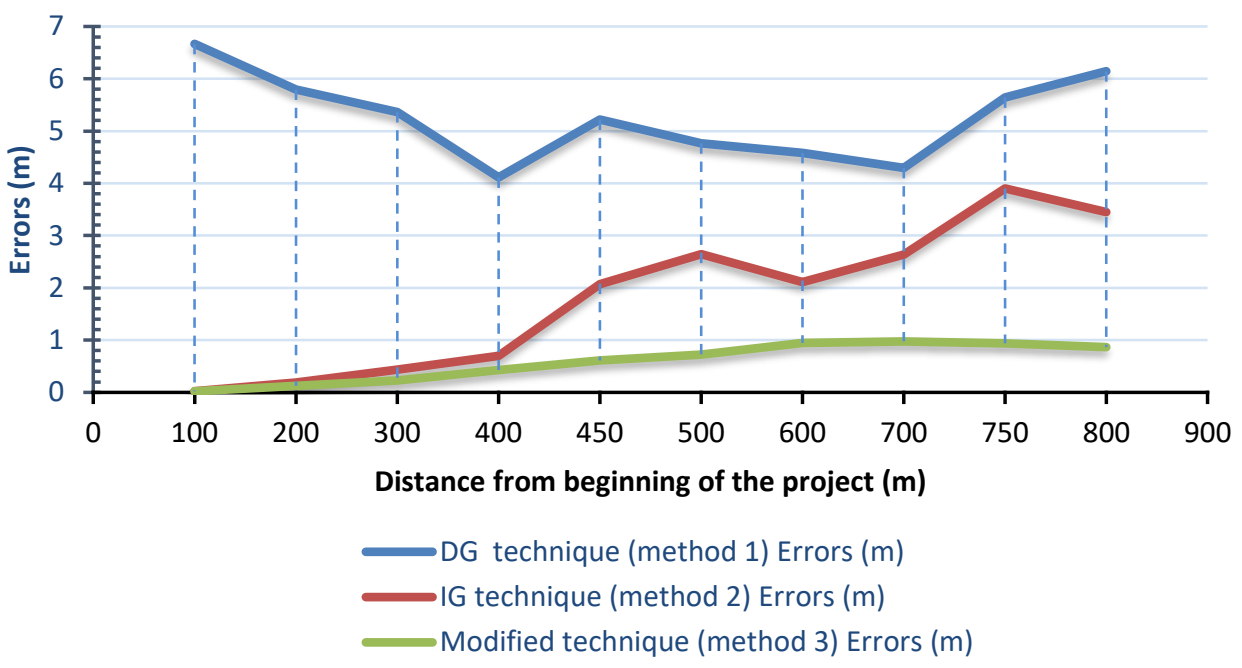

Fig. 5. The comparison of the various methods along with the UAV linear project.

From Table 5 and Figure 5, it can be seen that method 2 using indirect georeferencing technique, and method 3 using Modified georeferencing technique, the errors increased as they moved away from the 3 GCPs. This is due to the significant influence of the GCPs on the accuracy near them, and this influence decreases the farther away from them. On the other hand, the errors in direct georeferencing technique method 1 were like a wave from the beginning of the project to the end. This is due to factors affecting drone GPS accuracy and synchronization with image capture. It is also clear that method 3 is the most accurate. From the observation of the method 3 errors curve, the curve is in a relative escalation up like curve of method 2 to a distance of $250 \mathrm{~m}$ away from the start of the project. In addition, the influence of $3 \mathrm{GCPs}$ and enhanced camera stations positions work together to a distance of $600 \mathrm{~m}$ away from the start of the project. Then again, the curve was almost constant to the end of the project. This is due to the end of the significant influence of the 3 GCPs up to this distance, and then the influence of the enhanced camera stations positions begins to appear. The curve is almost constant because the errors of enhanced camera stations positions were almost constant in all locations of the project, and not affected near or far from the location of GCPs. Therefore, the curve was almost constant in the last part of the project. 


\subsection{Discussion}

In this study, a lower number of GCPs (three GCPs) were used and locally distributed in one place at the start of the linear UAV project. In the photogrammetric literature, the number of GCPs to be used in a conventional aerial manned survey has previously been mentioned. It is generally accepted that the higher the resulting precision is [12], the more control points are used. The costs involved in setting control points in large geographic areas, however, entail a compromise to ensure sufficient precision with low operational costs [12]. Although these standards should originally refer to UAV-based photogrammetry, this new technology has unique features for smaller sites and the increasing use of non-metric cameras and image simulation self-calibration. There is no published literature on how the number of GCPs influences the precision of a UAV SfM analysis, and several findings are either inconclusive or inconclusive even contradictory.

The purpose of this study was to obtain accurate digital models of linear objects from images from UAVs by refining the camera station positions and using a small number of locally located GCPs. The method of indirect georeferencing using ground control points is still more accurate than direct georeferencing without using any GCPs. In the locations that the use of GCPs is not easy to distribute along with linear projects. while the georeferencing accuracy needs to be improved by using multiple GCPs that are distributed along with the linear project and more accurate data from drone GPS. It is advisable to use the proposed method (The modified georeferencing technique) in hard-to-reach areas and areas closed for signal transmission from a satellite because it gives more accurate results than the indirect georeferencing method that uses the same locally few numbers of GCPs and raw data of drone GPS. In comparison to indirect georeferencing, the modified technology acquires no additional costs. It simply requires more office work, but the final result is well worth the effort.

Other studies [13] examined the capability of GNSS and IMU sensor integration data post-processing in UAVs to outperform DG with results of centimeter accuracy. [14] tiny aerial fixed-wing aircraft for specific corridors. [15] a VBS RTK GPS aided self-calibration package adjustment was evaluated for aerial triangulation of UAV images to update 1:5000 topographic maps; collected data by flying a fixed-wing UAS equipped with a $550 \mathrm{~m}$ Canon EOS 5D Mark II camera of $24 \mathrm{~mm}$ focal length. The Root Mean Square Error (RMSE) was $0.27 \mathrm{~m}$ in planimetry and $0.24 \mathrm{~m}$ in height accuracy. Using the modified technique described in this study in combination with the use of regular UAVs can produce results close to using direct georeferencing with an RTK-UAV. On the other hand, the modified technique is much less expensive compared to using direct georeferencing RTK-UAV.

\section{Conclusions}

From the results of this research, it is clear that the enhancement of the camera stations positions using the LR model has a clearer effect on the accuracy of georeferencing better than using the drone GPS imaging centers coordinates. The errors decreased from meters $(3.07 \mathrm{~m})$ to decimeters $(0.74 \mathrm{~m})$ when using the same 3 GCPs in the edge of the UAV linear project. In errors assessment, indirect georeferencing (method 2) using only 3 GCPs without using the enhanced camera stations positions, $\mathrm{Mx}, \mathrm{My}$, and $\mathrm{Mz}$ are respectively about 3.7, 2.9, and 4.7 times the modified georeferencing technique (method 3) using the enhanced camera stations positions and the same 3 GCPs in small part ( $2 \%$ of project length) in the edge of the project. Moreover, the enhanced camera stations positions in method 3 are more accurate than drone GPS data according to the differences between the computed data in the reference method and them. The RMSE of the difference between referenced and enhanced camera stations positions, DXo, DYo, and DZo are respectively about 6.0, 1.3, and 2.1 times 
the drone GPS data. The use of the modified georeferencing technique has a significant impact on the accuracy of georeferencing with no additional cost, especially in projects that GCPs cannot be distributed throughout the project.

\section{Acknowledgement}

We would like to thank State University of Land Use Planning, Moscow, Russia for providing the materials and laboratories used in this research. The researcher Amr M. Elsheshtawy is funded by a scholarship under the joint (Executive Program between Egypt and Russia)

\section{References}

1. Z. Jaal, J. Abdullah, User's Preferences of Highway Landscapes in Malaysia: A Review and Analysis of the Literature. Procedia - Soc. Behav. Sci. 36, 265-272 (2012).

2. M. M. S. Albattah, Optimum Highway Design and Site Location Using Spatial Geoinformatics Engineering. J. Remote Sens. GIS. 5, 10 (2016).

3. D. A. Cucci, M. Rehak, J. Skaloud, Bundle adjustment with raw inertial observations in UAV applications. ISPRS J. Photogramm. Remote Sens. 130, 1-12 (2017).

4. E. M. Amr, G. A. Larisa, E. A. Mohamed, in ACM International Conference Proceeding Series (2020).

5. I. Colomina, P. Molina, Unmanned aerial systems for photogrammetry and remote sensing: A review. ISPRS J. Photogramm. Remote Sens. 92, 79-97 (2014).

6. E. E. Elnima, A solution for exterior and relative orientation in photogrammetry, a genetic evolution approach. J. King Saud Univ. - Eng. Sci. 27, 108-113 (2015).

7. D. Turner, A. Lucieer, Z. Malenovský, D. H. King, S. A. Robinson, Spatial coregistration of ultra-high resolution visible, multispectral and thermal images acquired with a micro-UAV over Antarctic moss beds. Remote Sens. 6, 4003-4024 (2014).

8. J. Škaloud, M. Cramer, K. P. Schwarz, Exterior Orientation by Direct Measurement of Camera Position and Attitude. Int. Arch. Photogramm. Remote Sensing1. XXXI, 125-130 (1996).

9. M. Rehak, J. Skaloud, Time synchronization of consumer cameras on Micro Aerial Vehicles. ISPRS J. Photogramm. Remote Sens. 123, 114-123 (2017).

10. E. A. Mohamed, Y. G. Albert, E. M. Amr, in ACM International Conference Proceeding Series (2020).

11. A. M. Hamdy, M. A. Elshewy, A. M. Elsheshtawy, Enhancing and Applying the Accuracy of RTK-GNSS Elevations in Earthworks Estimation. 14, 24-31 (2020).

12. P. R. Wolf, B. A. Dewitt, B. E. Wilkinson, Elements of Photogrammetry with Applications in GIS (McGraw-Hill Education, 2014).

13. M. Bláha, H. Eisenbeiss, D. Grimm, P. Limpach, in Proceedings of the International Conference on Unmanned Aerial Vehicle in Geomatics (UAV-g) (Copernicus, 2011), vol. 38, pp. 131-136.

14. M. Rehak, J. Skaloud, Fixed-wing micro aerial vehicle for accurate corridor mapping. ISPRS Ann. Photogramm. Remote Sens. Spat. Inf. Sci. 2, 23-31 (2015).

15. S.-H. Chio, VBS RTK GPS-assisted self-calibration bundle adjustment for aerial triangulation of fixed-wing UAS images for updating topographic maps. Bol. Ciências Geodésicas. 22, 665-684 (2016). 